\title{
THE GEM PROJECT: AN INTERNATIONAL COLLABORATION TO SURVEY GALACTIC RADIATION EMISSION]
}

\author{
S. TORRES 2 , V. CAÑON, R. CASAS, A. UMAÑA \\ Observatorio Astronómico, Universidad Nacional de Colombia and Centro Internacional de Física, \\ Bogotá, Colombia \\ C. TELLO, T. VILLELA \\ INPE $/ C N P q$, Brazil \\ M. BERSANELLI \\ IFCTR-CNR, Milano, Italy \\ M. BENSADOUN, G. DE AMICI, M. LIMON, G. SMOOT, C. WITEBSKY \\ Lawrence Berkeley National Laboratory, UC Berkeley, CA, USA
}

\begin{abstract}
The GEM (Galactic Emission Mapping) project is an international collaboration established with the aim of surveying the full sky at long wavelengths with a multi-frequency radio telescope. A total of 745 hours of observation at $408 \mathrm{MHz}$ were completed from an Equatorial site in Colombia. The observations cover the celestial band $0^{h}<\alpha<24^{h}$, and $-24^{\circ} 22^{\prime}<\delta<+35^{\circ} 37^{\prime}$. Preliminary results of this partial survey will be discussed. A review of the instrumental setup and a $\sim 10^{\circ}$ resolution sky map at $408 \mathrm{MHz}$ is presented.
\end{abstract}

\section{The GEM Project}

Synchrotron radiation from relativistic electrons accelerated by the magnetic field of the Galaxy constitute the main component of diffuse galactic emission at low frequencies (300 $\mathrm{MHz}$ to few $\mathrm{GHz}$ ). At higher frequencies and high galactic latitude free-free (bremsstrahlung) emission from ionized hydrogen starts becoming the dominant component $(20-60 \mathrm{GHz})$ and beyond $60 \mathrm{GHz}$ interstellar dust emission begins to dominate. A precise measurement and mapping of the diffuse galactic emission at low frequencies can tell us much about the cosmic ray electrons and the dynamics of the Galaxy.

The discovery of cosmic microwave background (CMB) anisotropies (Smoot et al. 1992) and subsequent measurements at large angle scales (Hancock et al. 1994; Ganga et al. 1993) have underlined the importance of an accurate determination of temperature spectral indices $\left(T_{b} \propto \nu^{-\beta}\right)$ for the synchrotron and free-free mechanisms in the Galaxy. The existing surveys (Reich and Reich 1988; see also Table 1 of Lawson et al. 1987 for earlier work) are insufficient to provide the required accuracy as pointed out by Davies et al. (1996). This fact is due to the large zero level and gain uncertainties (i.e. $\pm 3 \mathrm{~K}$ and $10 \%$ for the $408 \mathrm{MHz}$ of Haslam et al. (1982) and $\pm 0.5 \mathrm{~K}$ and $0.5 \%$ for the $1420 \mathrm{MHz}$ survey of Reich and Reich (1988) respectively). Except for the Haslam et al. $408 \mathrm{MHz}$ survey, which is a composition of several patches made with four different telescopes, all others have partial sky coverage. The non-uniformity of

\footnotetext{
${ }^{1}$ Presented by S. Torres at the UN/ESA Workshop on Basic Space Sciences: From Small Telescopes to Space Missions, Colombo, Sri Lanka 11-13 January 1996

${ }^{2}$ e-mail: storres@uniandes.edu.co
} 
these data sets and residual striping effects constitute a serious limitations to the quality of the maps. Accurate, multifrequency data are needed in order to extrapolate the galactic emission at higher frequencies (Brandt et al. 1994; Masi et al. 1991), especially due to the fact that the synchrotron spectral index presents variations with galactic latitude (Lawson et al. 1987). Future CMB experiments conducted from satellites such as COBRAS/SAMBA and MAP will carry state of the art detectors but nevertheless will be limited by the accuracy of foreground emission removal. There is a well justified need for a full sky, homogeneous, multi-frequency, and accurately calibrated survey of the galactic radio continuum.

Besides producing crucial information for CMB observations, the GEM maps are scientifically important in themselves. The spectrum of galactic radio emission at long wavelengths is dominated by synchrotron radiation emitted by relativistic cosmic ray electrons accelerated by the large scale galactic magnetic field. The synchrotron power emitted by electrons depends on the electron energy density and the magnetic field intensity (Ginzburg \& Syrovatskii 1965). Thus, a survey of the radio emission provides useful information from which the galactic magnetic field and the cosmic ray electron energy spectrum can be studied. Knowledge derived from these studies have special relevance in models of cosmic ray acceleration.

Motivated by the above mentioned need to apply corrections to the galactic contamination present in cosmic microwave background (CMB) maps, an international collaboration was established to measure the galactic continuum emission in the range $408-5000 \mathrm{MHz}$ (De Amici et al. 1994). The GEM collaboration was started by groups in Brazil (INPE/CNPq), Colombia (CIF and Observatorio Astronómico), Italy (CNR), USA (Lawrence Berkeley National Lab/UCB), and later joined by Spain (IAC).

\section{Experimental Setup}

One of the major difficulties faced by a full sky radio survey is the need to achieve accurate calibration. The design strategy consists of a 'portable' radio-telescope that can be moved to sites at different latitudes. Using the same calibrated instrument allows for a consistent merging of patches of the sky taken at different sites. An additional advantage is that moving the instrument at different latitudes allows pointing the main beam at a small angle from the local zenith, thus minimizing the atmospheric absorption seen at large zenith angles (important at the higher frequencies). The GEM telescope is mounted on a rotating base and the pointing of the main beam is kept at a fixed angle from the zenith to keep the atmospheric contribution constant. The mechanical design of the mount system allows for changes in the zenith angle of the antenna. The antenna sidelobes can be checked by observing the same part of the sky at different zenith angles, therefore obtaining a direct measurement of ground contribution. The combined motion of the rotating base and the Earth's rotation results in a swath of the sky seen at each latitude. In principle one could cover the whole sky by collecting data in this mode from three equidistant latitudes (i.e. $60^{\circ} \mathrm{S}, 0^{\circ}$ and $60^{\circ} \mathrm{N}$ ). In practice it is desirable to allow for overlaps of the covered regions so as to ensure a self-consistent data set. We have acquired several hundred hours of observation at 408, 1465, 2300 and $5000 \mathrm{MHz}$ from an Equatorial site in Colombia and a Northern site in Bishop, California. The telescope is currently at the IAC-Tenerife Observatory in Spain. An overview of the experimental setup and preliminary results of the $408 \mathrm{MHz}$ survey conducted from the Equatorial site will be presented.

A block diagram (Fig. 1) shows the main parts that form the GEM system. The main section is made of the parabolic reflector, the feed antenna, the rotating base and the radiometer. Table 1 summarizes the instrumental parameters of GEM some of which have been computed as described below.

\subsection{Parabolic reflector, base and feed antenna}

GEM uses a Scientific Atlanta 5.5-m parabolic reflector mounted on an alt-azimuth rotating base. The 408, 1465 and $2300 \mathrm{MHz}$ receivers use a prime focus feed: a backfire helix at the low frequencies, and a conical antenna at $2300 \mathrm{MHz}$. The $5000 \mathrm{MHz}$ receiver unit and conical antennae are mounted at the Gregorian focus. Aluminum panels extend the parabolic reflector surface to a total diameter of 9.5$\mathrm{m}$. The purpose of this aluminum shield is to minimize diffracted ground emission and to allow us to 
Table 1: GEM parameters

\begin{tabular}{ll}
\hline Parameter & value \\
\hline \hline System & \\
Receiver frequencies & $408,1465,2300,5000 \mathrm{MHz}$ \\
Reflector diameter & $5.5 \mathrm{~m}$ \\
Reflector diameter with extension & $9.5 \mathrm{~m}$ \\
Postdetection integration time & $0.56 \mathrm{~s}$ \\
Base rotation speed (nominal) & $1 \mathrm{rpm}$ \\
\hline $408 \mathrm{MHz}$ Receiver & \\
System temperature & $104 \pm 6 \mathrm{~K}$ \\
Gain & $58 \pm 1 \mathrm{~K} \mathrm{~V}-1$ \\
Band width & $28 \mathrm{MHz}$ \\
Sensitivity & $26 \mathrm{mK} /$ integration time \\
Beam width (FWHM) & $11.3^{\circ}$ \\
Baseline susceptibility & $-3 \mathrm{~K}{ }^{\circ} \mathrm{C}^{-1}$ \\
Gain susceptibility & $-2.7 \times 10^{-4} \%{ }^{\circ} \mathrm{C}^{-1}$ \\
\hline
\end{tabular}

determine the beam efficiency and loss by covering up with highly reflective opaque material half the $4 \pi$ solid angle.

The back-fire helix feed antenna (408 MHz) consists of a 9.5 turns made of copper piping of $9.6 \mathrm{~mm}$ diameter. The turn length is $0.92 \lambda$, the spacing between turns is $15.4 \mathrm{~cm}$ and the axial length is 148 $\mathrm{cm}$. The feed antenna is sensitive to circularly polarized radiation. The main lobe width of the combined antenna/reflector assembly is obtained using the transit of the sun in front of GEM. A plot of the $408 \mathrm{MHz}$ signal voltage versus the separation angle between GEM's pointing and the sun shows the Gaussian-like shape of the main lobe with FWHM of $11.3^{\circ}$.

The mount assembly rests on a rotating base with a velocity of $1 \mathrm{rpm}$. An azimuth angle encoder mounted on the rotating axis of the main GEM assembly and a similar encoder on the horizontal axis provide a $0-10 \mathrm{~V}$ analog voltage proportional to the angle. The zero angle resulting from this reading is calibrated using the sun signal in the data in combination with the sun ephemerides. These calibration parameters were verified by checking GEM's mechanical orientation with respect to the geographic North employing a theodolite.

\subsection{The $408 \mathrm{MHz}$ receiver}

The $408 \mathrm{MHz}$ radiometer uses a total power receiver with two RF amplification stages and one DC amplification $(\times 1000)$ after detection (Fig. 2). A cavity filter $(\Delta \nu=28 \mathrm{MHz})$ at the front end of the receiver and a tubular filter after $\mathrm{RF}$ amplification are used.

The ambient temperature of the receiver is controlled and isolated from the outside temperature by warming the inside of the receiver box with resistance heaters while cooling the inside of a bigger box that encloses the receiver box. Temperature sensors and a regulating circuit keep the operating temperature of the receiver to within $\pm 0.2{ }^{\circ} \mathrm{C}$.

The susceptibility of the radiometer output voltage baseline to changes on operation temperature is measured as $-3 \mathrm{~K} /{ }^{\circ} \mathrm{C}$. Thus, within the temperature stability achieved one expects baseline drifts up to $0.6 \mathrm{~K}$ which typically occur in time scales of 8 to 9 hours. We correct for temperature induced baseline drifts by using the information from the various temperature sensors and the $\mathrm{K} /{ }^{\circ} \mathrm{C}$ slope quoted above. Gain changes are monitored by injecting a fixed amplitude reference pulse every 45 seconds. The reference noise pulse is generated by a diode and is connected to the input of the first amplifier stage via a directional coupler.

Radio frequency interference (RFI), due to local radio communications or electric discharges in the 
atmosphere can cause excessive dispersion of the observed signal. An RFI detection circuit signals the presence of such anomalous data. This circuit produces an output voltage $V_{\text {sat }}$ proportional to the number of times that the signal average crosses some preset threshold during the integration time. Cutting data above some $V_{\text {sat }}$ threshold effectively acts as a low-pass filter on the signal.

\subsection{Gain and system temperature calibration}

Gain, $G$, and system temperature, $T_{\text {sys }}$, are obtained by recording the radiometer signal voltage as the input is switched from a cold to a hot target. A $50 \Omega$ termination submerged in a bath of liquid Nitrogen (LN) at ambient pressure is used as a reference cold target. A similar termination at room temperature provides the warm reference target. The temperature of the warm target is monitored by a thermocouple while that of the cold target, $75 \mathrm{~K}$, is measured using a reference platinum resistance immersed in the LN. Including the contributions of the connectors and the attenuation and reflection properties of the coaxial cable (at $408 \mathrm{MHz}$ ) connecting the termination to the radiometer brings the equivalent $\mathrm{LN}$ temperature to $92 \pm 2 \mathrm{~K}$. The measured parameters are: $G=58 \pm 0.9 \pm 0.2 \mathrm{~K} \mathrm{~V}^{-1}, T_{\mathrm{sys}}=104 \pm 6 \pm 1 \mathrm{~K}$. The first error is systematic, the second statistical. Systematic errors are obtained with the error propagation formulas taking into account the errors in the measurements of target temperatures and signal voltages. The statistical errors quoted come from the standard deviation of the measurements done over a period of 1 hour. The sensitivity of the receiver implied by the measurement of $T_{\text {sys }}$ is $26 \mathrm{mK} /$ integration time and is consistent with the rms spread of the antenna temperature when looking at a fixed and known temperature target.

\subsection{Data acquisition system}

A 16 bit ADC samples 14 multiplexed analog channels (radiometer signal, angle encoders, temperature sensors, $V_{\text {sat }}$ and noise source voltage) and places the digitized information on a serial output. A time stamp and a sequential identifier number ('frame number') is added to the data stream. The time tag is the UT time provided by an external digital clock/receiver synchronized with the WWV station in Denver. Pulses from an internal clock $(100 \mathrm{~Hz})$ are divided down to provide the integration time of 0.56 seconds, which effectively defines the basic data rate of 50 bytes/s.

The data acquisition (DAQ) unit is mounted in a separate NIM crate box near the receiver whose DC output signal is connected via coaxial cable. The output of the DAQ unit is serial digital data sent to the DAQ computer in the control room through slip rings. The DAQ computer (a Macintosh Performa) performs a routine data quality check and stores the data on disk for future analysis.

\section{The Equatorial Site}

To complement the observations made or planned from California, Brazil and Spain an Equatorial site seemed to be the natural place to look. Colombia, in particular, is located on the Equator and has high peaks. However, the tropical weather conditions characteristic of equatorial latitudes can place severe restrictions. Site studies (Torres et al. 1992; Hoeneisen et al. 1992) in Colombia showed that there exist at least two dry seasons suitable for radio-astronomy and that there is a large variation of weather conditions at the different regions of the country. Due to the complex terrain there are micro-climates with very dry regions. The selected site in Villa de Leyva (Boyacá, Colombia) is a region characteristic for its dry atmosphere, specially during the dry season (December - April).

The GPS coordinates and altitude of the site are: LAT $=5^{\circ} 37^{\prime} 7.84^{\prime \prime} \pm 0.59^{\prime \prime} \mathrm{N}, \mathrm{LONG}=73^{\circ} 35^{\prime} 0.53^{\prime \prime} \pm$ $0.72^{\prime \prime} \mathrm{W}$, Altitude $=2,173 \pm 28$ m.a.s.l. The errors quoted are statistical while the systematic error is the $3.2^{\prime \prime}$ allowed by DOD. 


\section{Data Reduction}

Every 0.56 seconds the DAQ module sends via serial RS-232 a 14 words frame of data to the on-line computer. The off-line analysis software prepares the data for pixelization and applies cuts as follows: azimuth and elevation angles are calibrated; Julian and Sideral time of each measurement is computed; data points with the sun within $30^{\circ}$ of the beam are rejected; noise source pulses are subtracted from the data and used for gain corrections; strong RFI signals are extracted by binning short segments of data in azimuth and rejecting data that shows large deviations from the mean of its corresponding bin; a polynomial fit baseline is found and corrected for temperature induced drifts; finally, the radiometer signal is calibrated and pixelized. Further cleaning of the data is done on the pixelized set by rejecting points that show large deviations with respect to the mean temperature of their corresponding pixel.

A total of 1,116 hours of observation at $408 \mathrm{MHz}$ were completed from the Equatorial site from March 13 to May 13 (1995). Data runs with strong presence of RFI were rejected. Data with elevation angles different than $60^{\circ}$ were used for ground contribution studies. The remaining 745 hours of data were analyzed. From the selected data-set $40 \%$ is rejected as follows: $10.6 \%$ is sun contaminated, $6.3 \%$ is RFI contaminated, $3.8 \%$ is labeled 'anomalous' and is cut at the time of the baseline fit, $6.4 \%$ due to temperature sensors out of bounds, $3.5 \%$ RFI contaminated as signaled by $V_{\text {sat }}$, and the remaining goes into the other cuts.

\section{The GEM $408 \mathrm{MHz}$ sky map}

As data-base structure to store pixelized data we used the skycube pixelization scheme used by COBE (Torres et al. 1989, Torres 1995). This choice is dictated by the availability of several libraries and procedures to handle sky maps in this format and because it makes it easier to compare our maps with others. With the $408 \mathrm{MHz}$ beam size of $\sigma_{\text {beam }} \sim 8.38 \times 10^{-2}$ radians GEM can distinguish $\sim\left(2 / \sigma_{\text {beam }}\right)^{2} \approx$ 600 independent pixels in the sky. Thus, skycube pixelization level 5 with 1536 pixels seems appropriate.

The primary output of the analysis software is a sky map with a $54 \%$ sky coverage corresponding to the $60^{\circ}$ wide celestial band to which we have access from the Equatorial site. This band covers the sky region $0^{h}<\alpha<24^{h},-24^{\circ} 22^{\prime}<\delta<+35^{\circ} 37^{\prime}$ in right ascension and declination respectively. Fig. 3 is a rendering of this map in an equatorial celestial projection. The number of observations per pixel goes from 18 to 13,266 with a mean of 3,576 and a sigma of 2,188 .

The standard deviation of the temperature for each pixel is $\sim 2 \mathrm{~K}$, which is somewhat higher than expected from the measured sensitivity, indicating that there remains spurious effects not completely removed from the signal.

The zero level of the calibrated map needs corrections due to all possible known contributions not originating in the Galaxy. The integrated background of faint extragalactic background radio sources at $408 \mathrm{MHz}$ is $3.19 \mathrm{~K}$ (Lawson et al. 1987). The CMB temperature is $2.726 \pm 0.010 \mathrm{~K}$ (Mather et al. 1994), atmospheric emission at $408 \mathrm{MHz}$ is $\leq 1 \mathrm{~K}$. The contribution due to antenna and transmission line insertion loss and sidelobes pickup is estimated using data at several elevations and comparing data taken during the day with data taken at night (difference in ambient temperature $\sim 20{ }^{\circ} \mathrm{C}$ ). The combined contribution of all these factors amounts to $17 \pm 8 \mathrm{~K}$ and has been taken into account in the map in Fig. 3. The preliminary data presented here still has larger uncertainties than what is required for CMB foreground removal. Work towards a more accurate determination of the beam pattern, antenna insertion loss and calibration parameters is in progress. A preliminary comparison with the map of Haslam et al. was done by means of a pixel-to-pixel correlation between the GEM map and the same region of the sky in the Haslam et al. map degraded to the same angular resolution as GEM. The correlation coefficient is $98.3 \%$ which is highly significant. A more detailed comparison will be treated in a forthcoming paper.

The tolerable errors in long-wavelength surveys used in CMB work are quite stringent (Brandt et al. 1994). This fact is specially relevant when the 'subtraction technique' is used (i.e. measure the foregrounds in the region of the spectrum where they are strong, and extrapolate to the millimeter and sub-millimeter region where the $\mathrm{CMB}$ is measured). The rapid propagation of errors in the interpolation 
does not favor this method. However, a multi-frequency analysis (Masi et al. 1991) or a multi-frequency Wiener filtering analysis (Tegmark \& Efstathiou 1995) seem to be a more promising choice to remove foregrounds from CMB maps. Thus GEM multi-frequency data in the range $408-5000 \mathrm{MHz}$ combined with IRAS $100 \mu \mathrm{m}$, COBE-DMR (31.5, 53 and $90 \mathrm{GHz})$ and COBE-DIRBE $140 \mu \mathrm{m}$ data should be sufficient to separate the galactic foreground from CMB maps.

Acknowledgments. - This work was supported in part by the Director, Office of Energy Research, Office of High Energy and Nuclear Physics, Division of High Energy Physics of he U.S. Department of Energy under contract No. DE-AC03-76SF00098, the National Science Foundation under the International Joint Research NSF 9102295, and Calspace grant No. CS-63-94. Colciencias (projects 2228-05-007-92, and 2228-05-103-96) provided funds to support GEM operations in Colombia. We owe thanks to many persons who helped make this a succesful project. In particular we would like to thank H. Arias, L. M. Cruz, M. Fonsesca, J. Gibson, D. Heine, G. Holguín J. Larsen, D. Maino, E. Mattaini, A. Pantoja, L. Peña, E. Santambrogio, J. Yamada.

\section{REFERENCES}

Brandt, W. N. et al.: 1994, Astrophys. J. 424, 1.

Davies, R. D., et al.: 1996, Monthly Not. Roy. Astr. Soc. 278, 925.

De Amici, G., et al.: 1994, Astrophys. Space Sci. 214, 151.

Ganga, K., et al.: 1993, Astrophys. J. 410, L57.

Ginzburg, V. L. \& Syrovatskii, S. I.: 1965, Annu. Rev. Astron. Astrophys. 3, 297.

Hancock, S. et al.: 1994, Nature 367, 333.

Haslam C.G.T, et al.: 1982, Astr. Astroph. Suppl. Ser. 47, 1.

Hoeneisen, B. et al.: 1992, Nuclear Phys. B (Proc. Suppl.), 28B, 191.

Lawson, K. D., et al.: 1987, Monthly Not. Roy. Astr. Soc. 225, 307.

Masi, S., et al.: 1991, Astrophys. J. 366, L51.

Mather, J. C., et al.: 1994, Astrophys. J. 420, 439.

Reich, P. and Reich W.: 1988, Astron. Astrophys. 74, 7.

Smoot, G. F., et al.: 1992, Astrophys. J. 396, L1.

Tegmark, M. \& Efstathiou, G.: 1995, MPI-PhT/95-62 (preprint).

Torres, S., et al.: 1989, in Data Analysis in Astronomy III (eds V. di Gesu, L. Scarsi, and M.C. Maccarone) 40, 319 (New York: Plenum)

Torres, S.: 1995, Astrophys. Space Sci., 228, 309.

Torres, S., et al.: 1993, Rev. Col. de Física, 25, 23.

\section{FIGURE CAPTIONS}

Figure 1. Block diagram of the GEM system.

Figure 2. Diagram of the $408 \mathrm{MHz}$ receiver.

Figure 3. GEM $408 \mathrm{MHz}$ sky map contour levels in equatorial celestial coordinates.

The temperature levels go from 22 to $160 \mathrm{~K}$ in constant increments of $4 \mathrm{~K}$ up to $100 \mathrm{~K}$ where the increment changes to $20 \mathrm{~K}$. 